\title{
Anticancer effect of cucurbitacin B on MKN-45 cells via inhibition of the JAK2/STAT3 signaling pathway
}

\author{
YOU-LI XIE ${ }^{1}$, WEN-HUI TAO ${ }^{2}$, TI-XIONG YANG ${ }^{1}$ and JIAN-GUO QIAO ${ }^{1}$ \\ Departments of ${ }^{1}$ General Surgery and ${ }^{2}$ Gastroenterology, Zhongnan Hospital of Wuhan University, \\ Wuhan, Hubei 430071, P.R. China
}

Received July 15, 2015; Accepted August 11, 2016

DOI: $10.3892 /$ etm.2016.3670

\begin{abstract}
The aim of the present study was to investigate the effect of cucurbitacin B on MKN-45 gastric carcinoma cells. Cell proliferation was determined using a cell counting kit-8 assay, and commercial cell cycle and apoptosis analysis kits were used to determine the cell cycle by flow cytometry. The mRNA expression of genes which mediate cell cycle checkpoints and apoptosis was detected using reverse transcription-quantitative polymerase chain reaction, and a terminal deoxynucleotidyl transferase dUTP nick end labeling assay was used to determine apoptosis rate. Western blot analysis was used to detect the protein expression levels of JAK2/STAT3 signaling pathway-associated proteins. The presented data show that cucurbitacin B significantly inhibited the proliferation of MKN-45 cells in a dose- and time-dependent manner. In accordance with these findings, cucurbitacin B blocked the progression of the cell cycle from $\mathrm{G} 0 / \mathrm{G} 1$ to $\mathrm{S}$ phase, which was confirmed by the mRNA expression analysis. Cucurbitacin $\mathrm{B}$ treatment significantly suppressed the expression of cyclin D1, cyclin E, cyclin-dependent kinase 4 (CDK4) and CDK2, while increasing the expression of p27. Cucurbitacin B also promoted cell apoptosis, as was determined by TUNEL assay and evaluation of mRNA expression. Further experiments suggested that the beneficial effect of cucurbitacin B on blocking the proliferation and inducing the apoptosis of MKN-45 cells may have been associated with suppression of the JAK2/STAT3 signaling pathway. Thus, the present results indicate that cucurbitacin $\mathrm{B}$ suppresses proliferation and promoted apoptosis of MKN-45 cells, which may be mediated by inhibition of the JAK2/STAT3 signaling pathway. Cucurbitacin B therefore may warrant further investigation as a feasible therapy for gastric carcinoma.
\end{abstract}

Correspondence to: Dr Wen-Hui Tao, Department of Gastroenterology, Zhongnan Hospital of Wuhan University, 169 Donghu Road, Wuhan, Hubei 430071, P.R. China

E-mail: 1657573376@qq.com

Key words: cucurbitacin B, MKN-45 cells, proliferation, apoptosis

\section{Introduction}

Gastric cancer is a common malignancy and ranks the first among all gastrointestinal cancers in terms of incidence (1). Therapies for gastric cancer include surgery, radiotherapy and chemotherapy $(2,3)$. Due to absence of the specific symptoms, the majority of cases are diagnosed at middle to late disease stage. For these patients, systemic chemotherapy is usually used to improve life quality and increase overall survival in certain patients (4). However, only a limited effect is achieved by chemotherapy, with toxic and side effects $(5,6)$. Constant efforts are now made to investigate novel therapies against late-stage gastric cancer.

Cell apoptosis is programmed cell death mediated by various signal transduction pathways under certain physiological or pathological conditions, in which multiple genes are involved. As a major pathway to clear abnormally proliferating cells, cell apoptosis may be intentionally induced to treat cancers $(7,8)$. Chemotherapy medications combined with traditional Chinese medicine preparations may greatly enhance the therapeutic effect and prevent postoperative relapse and metastasis $(9,10)$. Traditional Chinese medicine preparations are recommended for those at the late stage without surgical indications to prolong the survival (10).

Signal transducer and activator of transcription 3 (STAT3) is a latent transcriptional factor in the cytoplasm that is regulated by Janus kinase (JAK) phosphorylation. STAT3 is persistently unregulated in various tumor cell lines, including hematologic cancers (11), epithelial malignancies (12), typical breast cancer (13), head and neck cancer (14), ovarian cancer (15), prostate cancer (16) and gastric cancer (17). STAT3 upregulates genes associated with the proliferation and survival of tumor cells, either directly or indirectly. JAK2/STAT3 is an important intracellular signal transduction pathway in gastric cancer which regulates the growth, differentiation and apoptosis of gastric cancer cells (18). Persistent STAT3 activation has been shown to be correlated with proliferation stimulation and apoptosis inhibition of gastric cancer cells (18).

Cucurbitacin B is a tetracyclic triterpenoid isolated from plants of Cucurbitaceae family and proved to possess anti-tumor, anti-chemocarcinogenic and anti-inflammatory effects $(19,20)$. In vivo and in vitro experiments have shown that cucurbitacin B inhibits the proliferation of lung cancer cells (21) and pancreatic cancer cells, and induces 
apoptosis (22). Although cucurbitacin B strongly inhibits the growth of numerous tumor cell types, its effect on MKN-45 cells is unknown. In the present study, cucurbitacin B was used to treat MKN-45 cells reaching the logarithmic phase of growth, and its effect on proliferation and apoptosis of MKN-45 cells was observed. The mechanism was also discussed.

\section{Materials and methods}

Materials. Cucurbitacin B (6199-67-3; 98\% purity determined by high-performance liquid chromatography analysis) was ordered from Shanghai Winherb Medical Technology Co., Ltd. (Shanghai, China). A Cell Counting Kit-8 (CCK-8; ER612) assay was obtained from Dojindo Molecular Technologies, Inc. (Kumamoto, Japan). Dimethyl sulfoxide (DMSO) was ordered from Sigma-Aldrich (D2650; Merck KGaA, Darmstadt, Germany). RPMI 1640 medium, trypsin-EDTA (1316929) and fetal bovine serum (FBS) were obtained from Gibco (10099; Thermo Fisher Scientific, Inc., Grand Island, NY, USA). A Cell Cycle and Apoptosis Analysis Kit (C1052) was purchased from Beyotime Institute of Biotechnology (Haimen, China). TRIzol $^{\circledR}$ (15596-026) was purchased from (Invitrogen; Thermo Fisher Scientific, Inc., Carlsbad, CA, USA). The antibodies used to recognize the total and phosphorylated forms of p-STAT3 (4113), STAT3 (12640S), p-JAK2 (8082S), JAK2 (3230) and GAPDH (2118) were ordered from Cell Signaling Technology, Inc. (Danvers, MA, USA). For the in vitro study, cucurbitacin B was dissolved in DMSO.

Cell culture. Gastric cancer MKN-45 cells (CBP60488) were ordered from CoBioer Biosciences Co., Ltd. (Nanjing, China) and cultured in RPMI 1640 medium containing 10\% FBS. After $24-48 \mathrm{~h}$ incubation at $37^{\circ} \mathrm{C}$ with $5 \% \mathrm{CO}_{2}$, logarithmic growth phase cells were digested using $0.25 \%$ trypsin. After calculating the cell number, the cells were seeded into plates at a density of $1 \times 10^{5}$ cells per well. The cells used in this study were collected between passages 4 and 10 .

Measurement of cell proliferation. Cell proliferation was determined using a CCK-8 assay according to the manufacturer's instructions. After the MKN-45 cells were grown to $80 \%$ confluency in 96 -well plates, they were subsequently incubated with $0.1,1$ or $10 \mu \mathrm{M}$ cucurbitacin B for 12,24 and $48 \mathrm{~h}$. After the treatment, $10 \mu \mathrm{l} \mathrm{CCK}-8$ solutions were added to each well, then the plate was incubated in a $37^{\circ} \mathrm{C}$ incubator for $2.5 \mathrm{~h}$. Cell proliferation was determined by measuring the optical density at $450 \mathrm{~nm}$ using a plate reader (BioTek Instruments, Inc., Winooski, VT, USA).

Cell cycle progression assays. Cell cycle progression was determined using a cell cycle and apoptosis analysis kit in accordance with the manufacturer's instructions and fluorescence-activated cell sorting using BD FACSVerse (BD Bioscience, San Jose, CA, USA). Upon reaching 70-80\% confluency in the six-well plates, the MKN-45 cells were incubated with cucurbitacin $\mathrm{B}(10 \mu \mathrm{M})$ for $24 \mathrm{~h}$ prior to analysis.

Reverse transcription-quantitative polymerase chain reaction $(R T-q P C R)$. Total RNA was isolated from MKN-45 cells using TRIzol reagent, and its yield and purity were spectrophotometrically estimated by the A260/A280 ratio, which was determined using a NanoDrop 2000c (Thermo Fisher Scientific, Inc.). RNA ( $2 \mu \mathrm{g}$ of each sample) was reverse transcribed into cDNA using oligo (dT) primers and the Transcriptor First Strand cDNA Synthesis kit (04896866001; Roche Diagnostics, Basel, Switzerland) according to the manufacturer's instructions. SYBR Green PCR Master Mix (04707516001; Roche Diagnostics) was then used to quantify PCR amplifications using a Light Cycler 480 instrument with designated software (version 1.5; Roche Diagnostics). Conditions for PCR were as follows: Initial denaturation at $94^{\circ} \mathrm{C}$ for 2 min, followed by 25-35 amplification cycles consisting of denaturation at $94^{\circ} \mathrm{C}$ for $40 \mathrm{sec}$, annealing at $58^{\circ} \mathrm{C}$ for $45 \mathrm{sec}$ and elongation at $72^{\circ} \mathrm{C}$ for $1 \mathrm{~min}$. The $2^{-\Delta \Delta \mathrm{Cq}}$ method was used to calculate the relative mRNA levels of each target gene (23). The target gene mRNA expression was normalized against the internal control GAPDH and expressed relative to the control group. The primer sequences used were as follows: Cyclin-dependent kinase 4 (CDK4), forward 5'-AATGTTGTC CGGCTGATGGA-3' and reverse 5'-ACTGGCGCATCAGAT CCTTG-3'; CDK2, forward 5'-AAATCCTCCTGGGCTGCA AAT-3' and reverse 5'-GCGAGTCACCATCTCAGCAA-3'; cyclin D1, forward 5'-GATGCCAACCTCCTCAACGA-3' and reverse 5'-ACTTCTGTTCCTCGCAGACC-3'; cyclin E, forward 5'-AGAGGAAGGCAAACGTGACC-3' and reverse 5'-GAGCCTCTGGATGGTGCAAT-3'; P27, forward 5'-TCC GGCTAACTCTGAGGACA-3' and reverse 5'-AAGAATCGT CGGTTGCAGGT-3'; B-cell lymphoma 2 (Bcl-2), forward 5'-GTCATGTGTGTGGAGAGCGT-3' and reverse 5'-GAA ATCAAACAGAGGCCGCA-3'; Bcl-2-associated protein X (Bax), forward 5'-TCCACCAAGAAGCTGAGCGAG-3' and reverse 5'-GTCCAGCCCATGATGGTTCT-3'. GAPDH, forward 5'-GTCAAGGCTGAGAACGGGAA-3' and reverse 5'-TGGACTCCACGACGTACTCA-3'.

Western blot analysis. Cells were lysed in RIPA Lysis Buffer (G2002; Wuhan Google Biological Technology Co., Ltd., Wuhan, China) containing $50 \mathrm{mM}$ Tris- $\mathrm{Hcl}, 150 \mathrm{mM} \mathrm{NaCl}$, $1 \%$ Triton $\mathrm{X}-100,1 \%$ sodium deoxycholate and $0.1 \%$ SDS, which were then scraped into $1.5-\mathrm{ml}$ centrifuge tubes. The cell suspension centrifuged at $4^{\circ} \mathrm{C}$ for $30 \mathrm{~min}$ at $3,362 \times \mathrm{g}$, and the protein concentration was determined using a bicinchoninic acid protein assay kit (23227; Thermo Fisher Scientific, Inc.). A total of $20 \mu \mathrm{g}$ protein extract was used for SDS-PAGE, blotted onto immobilon-FL transfer membranes (EMD Millipore, Billerica, MA, USA), blocked with 5\% non-fat milk for 2 , then probed with the relevant antibodies (dilution, 1:1,000) overnight with gentle shaking at $4^{\circ} \mathrm{C}$. The goat anti-rabbit IRdye $800 \mathrm{CW}$ (cat. no. 926-32211) IgG and goat anti-mouse IRdye $800 \mathrm{CW}$ (cat. no. 926-32210) secondary antibodies (both purchased from LI-COR, Lincoln, NE, USA) were used at 1:10,000 dilution and incuvated at $37^{\circ} \mathrm{C}$ in Odyssey blocking for $1 \mathrm{~h}$. The protein expression was quantified using the Odyssey infrared imaging system (Li-COR Biosciences, Lincoln, NE, USA) and protein expression levels were normalized against the GAPDH internal control in the total cell lysate.

Terminal deoxynucleotidyl transferase dUTP nick end labeling (TUNEL) assay. TUNEL was performed using the 
in situ Cell Death Detection kit (11684795910; Roche Diagnostics), according to the manufacturer's instructions. The cells were grown on cover slips in a 24-well plate, which were fixed in 4\% paraformaldehyde (30525-89-4; Amresco, LLC, Cleveland, OH, USA) and permeabilized using $0.1 \%$ Triton X-100 (9002-93-1; Amresco, LLC) after treatment. The cells were incubated in $13 \mu \mathrm{l}$ (per cover slip) TUNEL reaction mixture (Roche Diagnostics) at $37^{\circ} \mathrm{C}$ for $1 \mathrm{~h}$. The nuclei were labeled with $13 \mu 1$ (per cover slip) 4',6-diamidino-2-phenylindole (DAPI; S36939; Invitrogen; Thermo Fisher Scientific, Inc.) for $30 \mathrm{sec}$, and DNA fragmentation was quantified using an IX51 microscope (Olympus Corporation, Tokyo, Japan) at $\mathrm{x} 200$. The percentages of TUNEL-positive cells relative to the DAPI-positive cells were counted by an investigator in a blinded-manner. Image analysis was performed using Image Pro-Plus software, version 6.0 (Media Cybernetics, Inc., Silver Spring, MD, USA).

Statistical analysis. All data are expressed as the mean \pm standard deviation. Differences between the groups were determined via one-way analysis of variance followed by a Student-Newman-Keuls test. $\mathrm{P}<0.05$ was considered to indicate a statistically significant difference.

\section{Results}

Cucurbitacin B inhibits proliferation of MKN-45 cells. To determine the effect of cucurbitacin $\mathrm{B}$ on MKN-45 cell proliferation, the effect of various doses of cucurbitacin B $(0.1-10 \mu \mathrm{M})$ over 12,24 and $48 \mathrm{~h}$ was investigated using a CCK-8 assay. Compared with the control, the proliferation of MKN-45 cells was significantly inhibited in a concentration-dependent manner, and the greatest level of proliferation suppression was induced by cucurbitacin $\mathrm{B}$ at a concentration of $10 \mu \mathrm{M}$ (Fig. 1). Results also showed that cucurbitacin B demonstrated a time-dependent inhibition of cell proliferation (Fig. 1).

Cucurbitacin B inhibits cell cycle progression from G0/G1 to $S$ phase. The effect of cucurbitacin B on cell cycle progression was analyzed using flow cytometric analysis. The control group showed an increased percentage of cells in S phase and decreased G0/G1 populations, while the cucurbitacin B-treated cells showed a suppression of cell cycle progression. Cucurbitacin B at a dose of $10 \mu \mathrm{M}$ reduced the percentage of cells in $\mathrm{S}$ phase and increased the G0/G1 populations compared with the control group (Fig. 2), suggesting that cucurbitacin B affected the $\mathrm{G} 0 / \mathrm{G} 1$ to $\mathrm{S}$ phase transition rather than being involved in the $\mathrm{S}$ or $\mathrm{G} 2 / \mathrm{M}$ phases.

Cucurbitacin B upregulates p27 and downregulates CDK4, $C D K 2$, cyclin D1 and cyclin E mRNA expression. To further characterize the potential mechanism underlying cucurbitacin B-induced cell cycle arrest, the effects of cucurbitacin B on cell cycle-associated genes, including cyclins, CDKs and cell cycle inhibitory expression, were analyzed using RT-qPCR. The results demonstrated that the mRNA expression levels of CDK4, CDK2, cyclin D1 and Cyclin E were significantly decreased in the $10 \mu \mathrm{M}$ cucurbitacin $\mathrm{B}$ group compared with the control group $(\mathrm{P}<0.01 ;$ Fig. 3$)$.

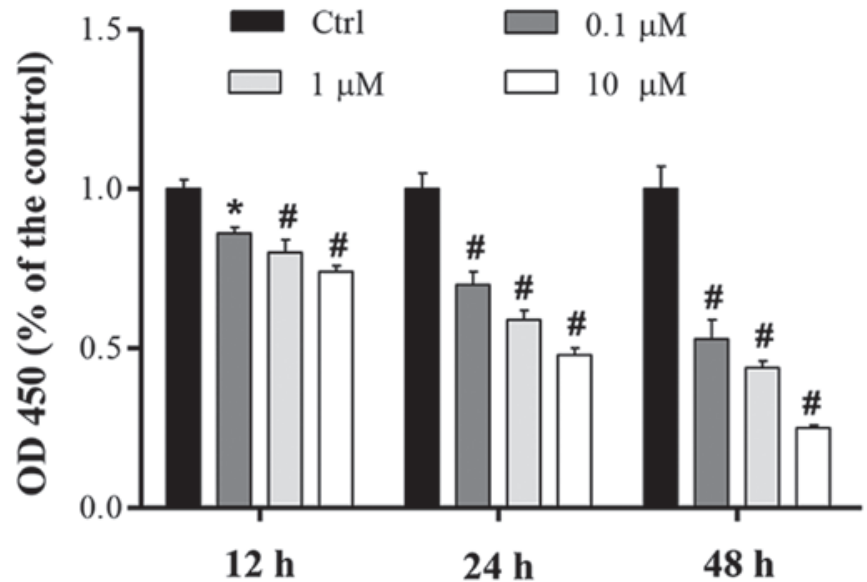

Figure 1. Effect of cucurbitacin B on proliferation of MKN-45 cells. MKN-45 cells were incubated with cucurbitacin B $(0.1,1$ or $10 \mu \mathrm{M})$ for 12,24 or $48 \mathrm{~h}$, and cell proliferation was examined by CCK-8 assay. Data are expressed as the mean \pm standard deviation. ${ }^{*} \mathrm{P}<0.05,{ }^{\#} \mathrm{P}<0.01$ vs. control group $(\mathrm{n}=5)$. OD, optical density; Ctrl, control.

CDK activity is additionally regulated by small proteins such as p27, which inactivates the cyclin-CDK complexes in the G1 phase, leading to cell cycle arrest. The present results demonstrated that the mRNA expression level of p27 in control group was low, whereas it was significantly increased by co-treatment with cucurbitacin $\mathrm{B}(\mathrm{P}<0.01$; Fig. 3$)$.

Cucurbitacin B induces apoptosis of MKN-45 cells. To investigate the effect of cucurbitacin B on MKN-45 cells apoptosis, a TUNEL assay was used to stain the apoptotic nuclei. Compared with the control group, a noticeable increase of cell apoptosis was observed in MKN-45 cells, which was significantly promoted by co-treatment with $0.1,1$ and $10 \mu \mathrm{M}$ cucurbitacin B for $24 \mathrm{~h}$ in a concentration-dependent manner. The highest level of apoptosis promotion was induced by cucurbitacin B at a concentration of $10 \mu \mathrm{M}(\mathrm{P}<0.01$; Fig. 4).

Cucurbitacin B regulates JAK2/STAT3 signaling pathway. To investigate the molecular mechanisms underlying the anti-proliferation and proapoptotic effects of cucurbitacin B, JAK2/STAT3 signaling pathway expression was evaluated. The activation of JAK2/STAT3 was assessed using western blot analysis. The results indicate that the phosphorylation of JAK2/STAT3 was significantly reduced by $0.1,1$ and $10 \mu \mathrm{M}$ cucurbitacin B, in a concentration-dependent manner (Fig. 5).

Cucurbitacin B promotes the mRNA expression levels of Bax and reduced those of $B c l-2$. To investigate the molecular mechanisms underlying the proapoptotic effects of cucurbitacin B, the apoptotic pathway was examined. The mRNA expression of the antiapoptotic protein $\mathrm{Bcl}-2$ was decreased, while the expression of the proapoptotic protein Bax was increased $(\mathrm{P}<0.01)$. These results were consistent with the finding that cucurbitacin B induced MKN-45 cell apoptosis (Fig. 6).

\section{Discussion}

The results of the present study demonstrated that cucurbitacin $\mathrm{B}$ decreased MKN-45 cell proliferation in a 


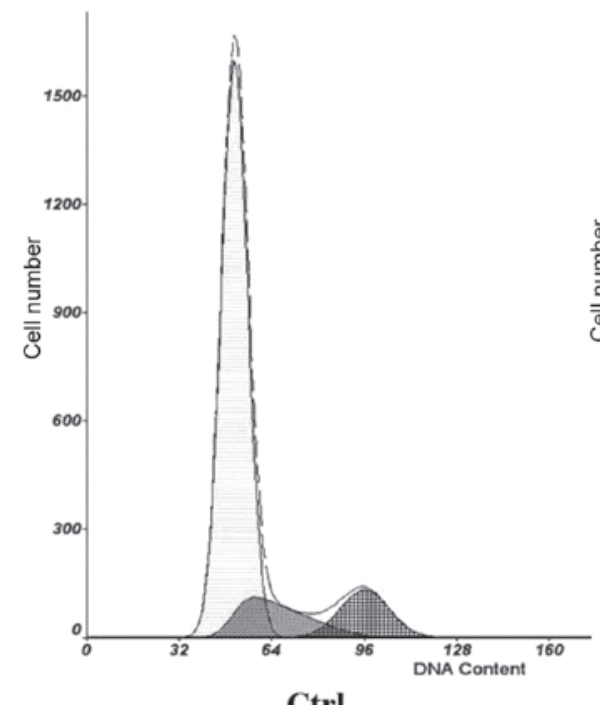

Ctrl

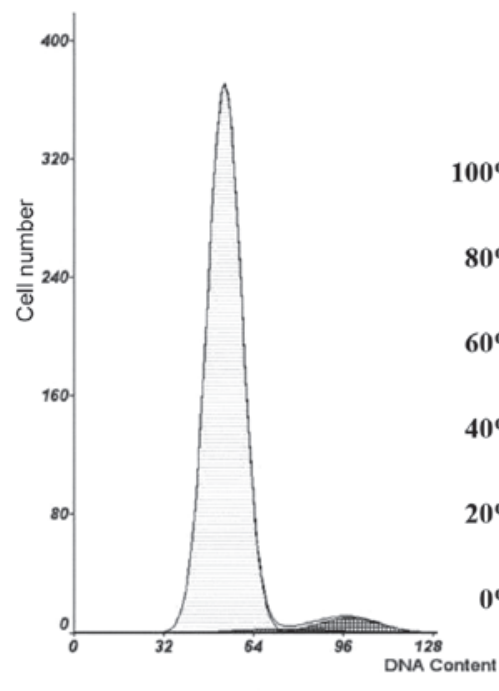

$10 \mu \mathrm{M}$

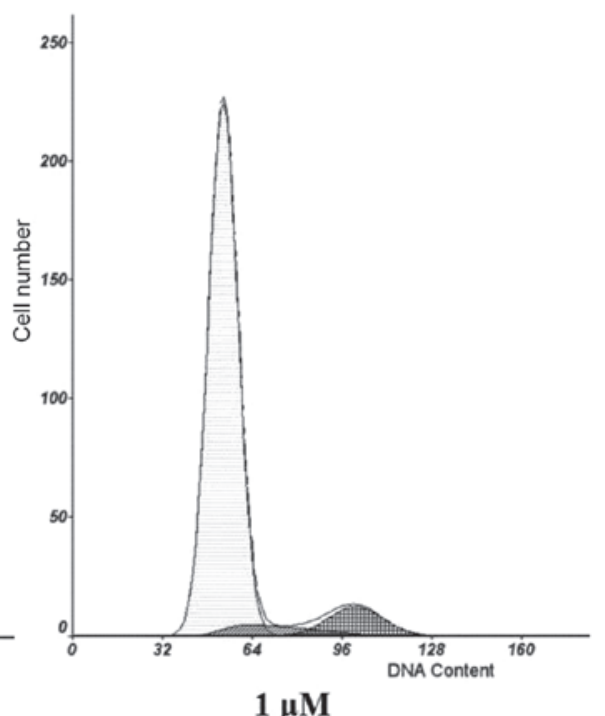

$1 \mu \mathrm{M}$

Figure 2. Effect of cucurbitacin B on cell cycle progression. MKN-45 cells were grown without or with cucurbitacin B (1 or $10 \mu \mathrm{M})$ for 24 h. PI fluorescence in MKN-45 cells was analyzed by flow cytometry, quantification of MKN-45 cells in the G0/G1, S and G2/M phases was also determined by flow cytometric analysis ( $\mathrm{n}=3$ ). PI, propidium iodide; Ctrl, control.

concentration-dependent manner. It was also found that cucurbitacin B suppressed the MKN-45 cell cycle at the G0/G1 to $S$ phase by inhibiting the mRNA expression of CDK4, CDK2, cyclin D1 and cyclin E, and increasing that of p27. Furthermore, the apoptosis rate of the MKN-45 cells was increased by cucurbitacin B in a concentration-dependent manner, which was verified by TUNEL staining. This result was further suggested by the inhibition of Bcl-2 and increase of Bax mRNA expression levels. These beneficial effects of cucurbitacin $\mathrm{B}$ on the MKN-45 cells were associated with the inhibition of the JAK2/STAT3 signaling pathway. Thus, the present study revealed the anticancer effects of cucurbitacin B on gastric cancer cells, which may lead to a novel strategy for gastric carcinoma treatment.

Cell proliferation is an important process for cell survival, which is also an important biological characteristic of tumor formation (13). Therefore, the inhibition of tumor proliferation is a crucial aim of tumor treatment. The present study found that cucurbitacin B was able to reduce viable cells in vitro in

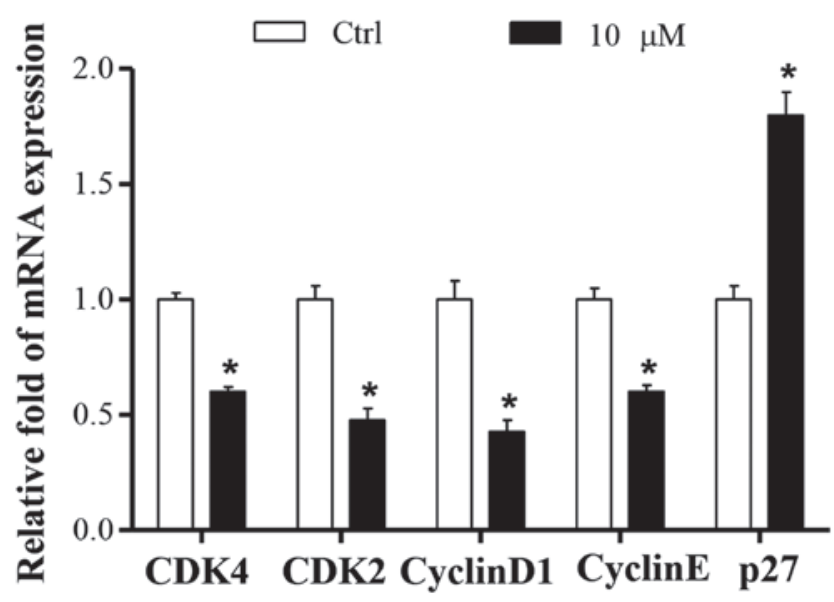

Figure 3. Effect of cucurbitacin B on the expression of multiple cell cycle regulatory genes in $\mathrm{MKN}-45$ cells. $\mathrm{MKN}-45$ cells were treated without or with cucurbitacin B $(10 \mu \mathrm{M})$ for $24 \mathrm{~h}$ and mRNA levels of the multiple cell cycle regulatory genes were analyzed. Experiments were performed in triplicate and repeated at least three times. $\mathrm{P}<0.01$ vs. control group $(n=3)$. CDK, cyclin-dependent kinase; Ctrl, control. 
A
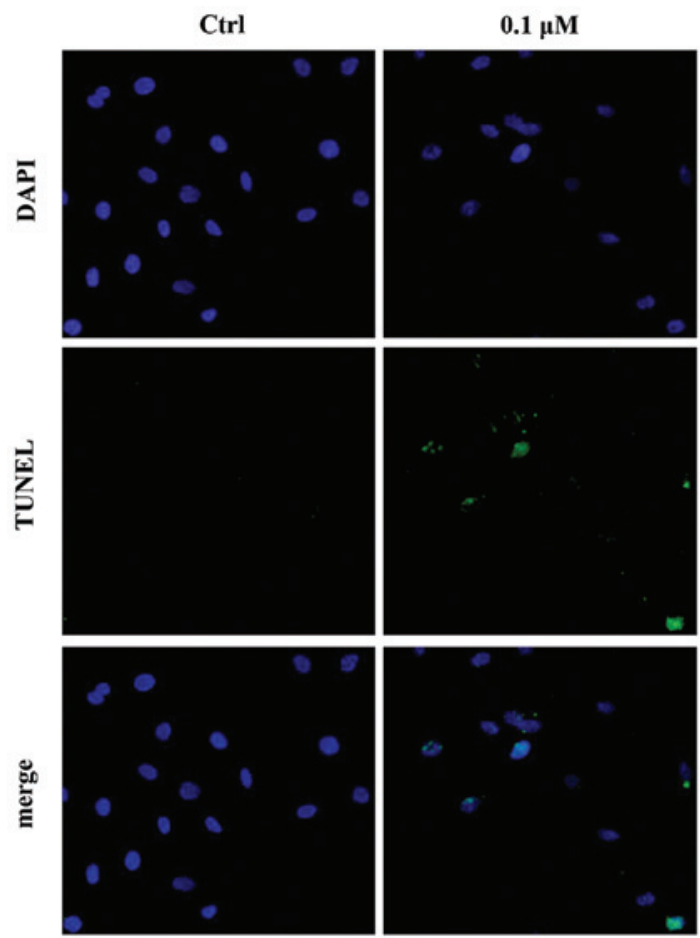

B

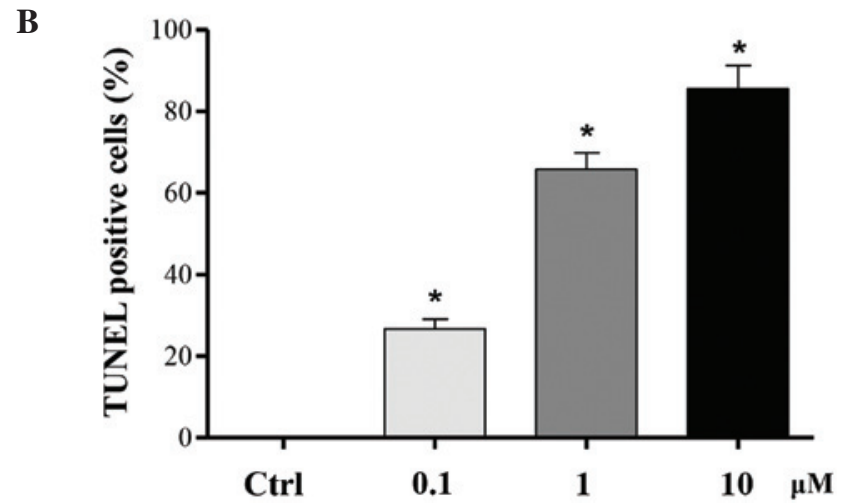

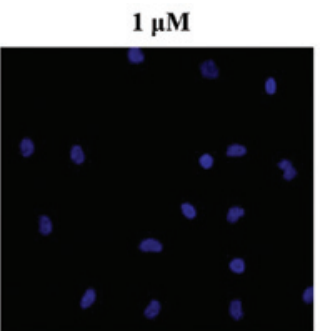
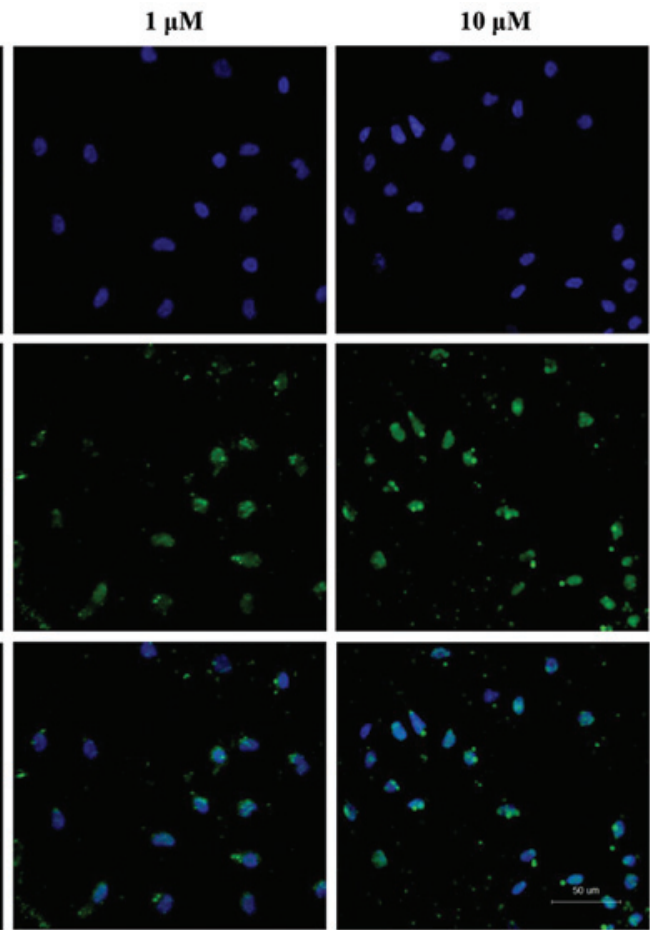

Figure 4. Effect of cucurbitacin B on apoptosis of MKN-45 cells. MKN-45 cells were treated with cucurbitacin B ( 0.1 , 1 or $10 \mu \mathrm{M})$ for 24 h, and cell apoptosis was examined by TUNEL assay. (A) Representative images and (B) quantitative results in MKN-45 cells. Data are expressed as the mean \pm standard deviation. ${ }^{*} \mathrm{P}<0.01$ vs. control group $(\mathrm{n}=6)$. TUNEL, terminal deoxynucleotidyl transferase dUTP nick end labeling; Ctrl, control.

a dose-dependent manner, and that the underlying mechanism involved cell cycle arrest. Following cucurbitacin B treatment, the percentage of cells in the G0/G1 phase was markedly increased.

Cell cycle checkpoints are control mechanisms which ensure proper DNA replication and chromosome division (24). Specifically, they are negative feedback mechanisms, which are activated in case of abnormal cell cycle events, such as DNA damage or obstructed DNA replication, to induce cell cycle arrest (25). The molecules driving the cell cycle are CDK/cyclin complexes, which contribute to the binding of cyclins to CDKs and show the activity of protein kinase $(24,25)$. In these complexes, cyclins function as the regulatory subunit, and CDKs as the catalytic subunit. The activity of CDKs may be inhibited by CDK inhibitors (CKIs) $(24,25)$. CDK4 and CDK2 are known to form complexes with cyclin D1 and cyclin E, which are essential for the regulation of cell cycle progression from the G0/G1 to $\mathrm{S}$ phase $(26,27)$. Another regulator controlling cell cycle progression is the CKI of p27, which forms heterotrimeric complexes with CDKs/cyclins to inhibit their activity, such as cyclin D1-CDK4 and cyclin E-CDK2 (28). In the present study, the expression of cell cycle regulatory genes in response to cucurbitacin B in MKN-45 cells was investigated. Cucurbitacin $B$ reduced the mRNA expression levels of cyclin D1, cyclin E, CDK4 and CDK2. Consistent with these changes, the mRNA expression of p27 was increased. These observations suggest that the antiproliferative activity of cucurbitacin B has a multifaceted effect on numerous target molecules critically involved in growth inhibition.

Tumor cells possess unlimited replication potential; and the occurrence of tumors is associated with the abnormal proliferation and differentiation of cells in addition to abnormal apoptosis (8). Loss of tumor cells via apoptosis is considered to be a contributing factor in tumor therapy and cancer molecular biology (8). There are two major apoptotic pathways, initiated by caspase- 9 and caspase- 8 respectively, which can activate caspase cascades (29). Apoptosis triggered by activation of the mitochondria-dependent caspase pathway represents 
A

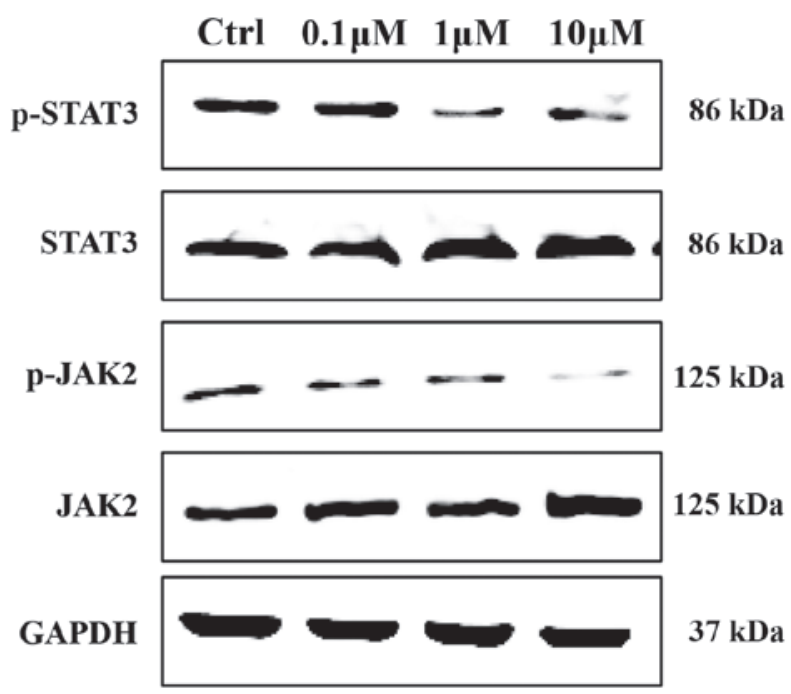

B

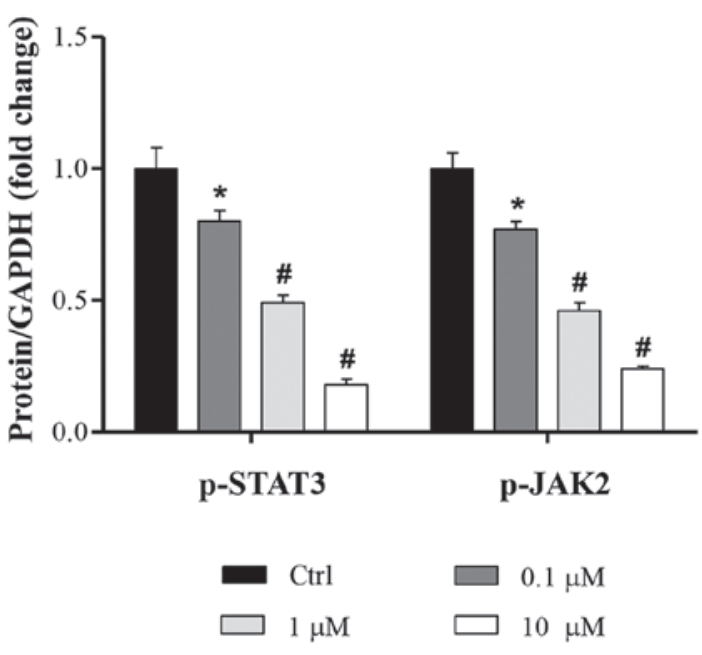

Figure 5. Effect of cucurbitacin B on activation of JAK2/STAT3 signaling pathway. MKN-45 cells were treated with 0.1 , 1 or $10 \mu \mathrm{M}$ cucurbitacin B for 24 h. Phosphorylation of JAK2 and STAT3 was analyzed by western blot analysis. (A) Representative western blot analysis and (B) quantitative results. Experiments were repeated at least three times. Data are expressed as the mean \pm standard deviation. ${ }^{*} \mathrm{P}<0.05$, ${ }^{\text {P }}<0.01$ vs. control group. p-, phosphorylated; STAT3, signal transducer and activator of transcription 3; JAK2, Janus kinase 2; GAPDH, glyceraldehyde-3-phosphate dehydrogenase; Ctrl, control.

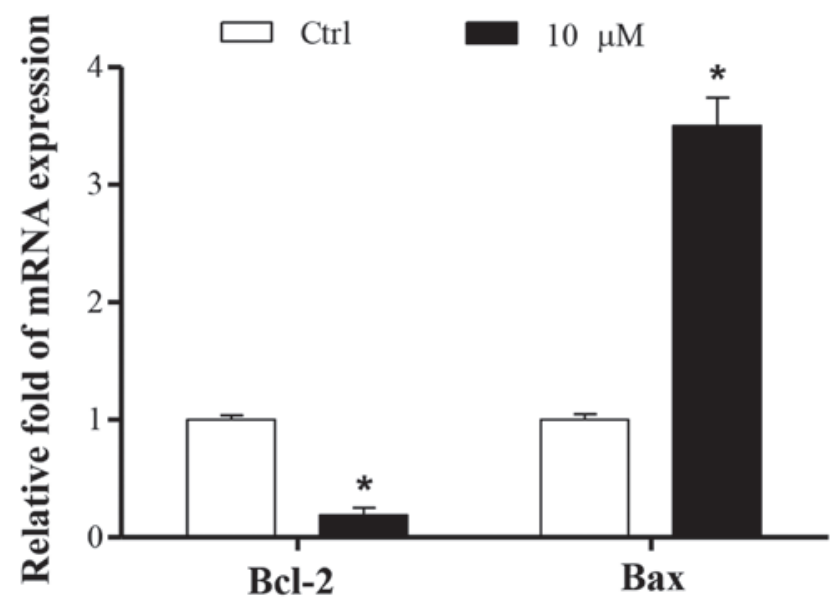

Figure 6. Effect of cucurbitacin B on the expression of multiple cell apoptosis genes in MKN-45 cells. MKN-45 cells were treated with cucurbitacin B $(10 \mu \mathrm{M})$ for $24 \mathrm{~h}$ and mRNA levels of the multiple cell apoptosis regulatory genes were analyzed. Experiments were performed in triplicate and repeated for at least three times. ${ }^{*} \mathrm{P}<0.01$ vs. control group $(\mathrm{n}=3)$. Ctrl, control; Bcl-2, B-cell lymphoma 2; Bax, Bcl-2-associated protein X.

the primary programmed cell death mechanism (29). This is activated by various intracellular stresses that induce permeabilization of the mitochondrial membrane (30). The Bcl-2 family consists of apoptosis regulators which serve a crucial function in the mitochondrial pathway of apoptosis. Various precipitating factors alter the intracellular expression of Bcl-2 family or protein structure (30). Through regulating permeability of the mitochondrial membrane, particularly the outer mitochondrial membrane, members of the Bcl-2 family induce the release of mitochondrial intermembrane space proteins (31). In the present study, cucurbitacin B downregulated the mRNA expression levels of Bcl-2 and upregulated the expression of Bax in the MKN-45 cells, which may regulate the proapoptotic effect of cucurbitacin $B$, suggesting that cucurbitacin B induced the apoptosis via the endogenous apoptosis pathway. These findings indicated that through its proapoptotic property, cucurbitacin B may be beneficial in gastric cancer treatment.

JAK/STAT pathway plays a crucial role in numerous signal transductions in vivo. The JAKs activation has an important role in cell differentiation, proliferation, apoptosis and migration (18). Structure activation of JAKs contributes to phosphorylation of the STAT family. STAT3 is a member of STAT family, which has been intensively studied in recent years (13-17). As confirmed by cell culture and animal models, the STAT3 signaling pathway provides an effective molecular target in the treatment of various human cancers (13-17). STAT3 signal is present in the epithelial cells of normal gastric mucosa and human gastric cancer cells (17). STAT3 activation mediates normal physiological processes of gastric mucosa (32). Overactivation of STAT3 is found in various types of gastric cancer cells (33). Pathological detection of gastric cancer indicated that STAT3 protein expression was obviously higher than that in normal gastric mucosa, the former being 2.14 times of that of the latter on average $(34,35)$. STAT3 protein expression in mildly differentiated gastric cancer was generally higher than that in highly differentiated gastric cancer $(34,35)$.

It has been shown that cucurbitacin B has potent antitumor activity against human pancreatic cancer cells by inhibiting JAK/STAT signaling pathway (36), and may also inhibit growth and induce apoptosis through JAK2/STAT3 signaling in SH-SY5Y human neuroblastoma cells (37). Therefore, we further aimed to determine whether the JAK2/STAT3 signaling pathway could be affected by cucurbitacin B. It was found that cucurbitacin B directly inhibited the phosphorylation of JAK2 and the downstream STAT3.

In conclusion, the present study demonstrated for the first time that cucurbitacin B inhibits the proliferation and promotes apoptosis of MKN-45 cells in vitro. These beneficial effects of cucurbitacin $\mathrm{B}$ on the MKN-45 cells were associated with the inhibition of the JAK2/STAT3 signaling pathway. The results 
of this study suggest that the use of cucurbitacin B to treat gastric carcinoma diseases warrants further investigation.

\section{References}

1. Allum WH, Blazeby JM, Griffin SM, Cunningham D, Jankowski JA and Wong R; Association of Upper Gastrointestinal Surgeons of Great Britain and Ireland, the British Society of Gastroenterology and the British Association of Surgical Oncology: Guidelines for the management of oesophageal and gastric cancer. Gut 60: 1449-1472, 2011.

2. Bernini M and Bencini L: Multimodal treatment of gastric cancer: Surgery, chemotherapy, radiotherapy and timing. Int J Surg Oncol 2012: 246290, 2012.

3. Yu C, Yu R, Zhu W, Song Y and Li T: Intensity-modulated radiotherapy combined with chemotherapy for the treatment of gastric cancer patients after standard D1/D2 surgery. J Cancer Res Clin Oncol 138: 255-259, 2012.

4. Roukos DH: Current advances and changes in treatment strategy may improve survival and quality of life in patients with potentially curable gastric cancer. Ann Surg Oncol 6: 46-56, 1999.

5. Xu CD: Clinical study of nimotuzumab combined with chemotherapy in the treatment of late stage gastric cancer. Asian Pac J Cancer Prev 15: 10273-10276, 2014.

6. Gao SR, Li LM, Xia HP, Wang GM, Xu HY and Wang AR Clinical observation on recombinant human endostatin combined with chemotherapy for advanced gastrointestinal cancer. Asian Pac J Cancer Prev 16: 4037-4040, 2015.

7. Zhang X, Zhong L, Liu BZ, Gao YJ, Gao YM and Hu XX: Effect of GINS2 on proliferation and apoptosis in leukemic cell line. Int J Med Sci 10: 1795-1804, 2013.

8. Yu YJ, Li YM, Hou XD, Guo C, Cao N and Jiao ZY: Effect of tissue factor on invasion inhibition and apoptosis inducing effect of oxaliplatin in human gastric cancer cell. Asian Pac J Cancer Prev 13: $1845-1849,2012$

9. Yan B, Liu L, Zhao Y, Xiu LJ, Sun DZ, Liu X, Lu Y, Shi J,Zhang YC, Li YJ, et al: Xiaotan Sanjie decoction attenuates tumor angiogenesis by manipulating Notch-1-regulated proliferation of gastric cancer stem-like cells. World J Gastroenterol 20: 13105-13118, 2014.

10. Zhu Y,Liu Y, Qian Y, Dai X, Yang L, Chen J, Guo S and Hisamitsu T: Research on the efficacy of Celastrus orbiculatus in suppressing TGF- $\beta 1$-induced epithelial-mesenchymal transition by inhibiting HSP27 and TNF- $\alpha$-induced NF- $\kappa$ B/ Snail signaling pathway in human gastric adenocarcinoma. BMC Complement Altern Med 14: 433, 2014.

11. Hung MH, Tai WT, Shiau CW and Chen KF: Downregulation of signal transducer and activator of transcription 3 by sorafenib: A novel mechanism for hepatocellular carcinoma therapy. World J Gastroenterol 20: 15269-15274, 2014.

12. Liu H, Ren G, Wang T, Chen Y, Gong C, Bai Y, Wang B, Qi H, Shen J, Zhu L, et al: Aberrantly expressed Fra-1 by IL-6/STAT3 transactivation promotes colorectal cancer aggressiveness through epithelial-mesenchymal transition. Carcinogenesis 36: 459-468, 2015.

13. Yao X, Liu H, Zhang X, Zhang L, Li X, Wang C and Sun S: Cell surface GRP78 accelerated breast cancer cell proliferation and migration by activating STAT3. PLoS One 10: e0125634, 2015.

14. Peyser ND, Freilino M, Wang L, Zeng Y, Li H, Johnson DE and Grandis JR: Frequent promoter hypermethylation of PTPRT increases STAT3 activation and sensitivity to STAT3 inhibition in head and neck cancer. Oncogene, 2015.

15. Wen W, Wu J, Liu L, Tian Y, Buettner R, Hsieh MY, Horne D, Dellinger TH, Han ES, Jove R and Yim JH: Synergistic anti-tumor effect of combined inhibition of EGFR and JAK/STAT3 pathways in human ovarian cancer. Mol Cancer 14: 100, 2015.

16. Hossain DM, Pal SK, Moreira D, Duttagupta P, Zhang Q, Won H, Jones J, D'Apuzzo M, Forman S and Kortylewski M: TLR9-targeted STAT3 silencing abrogates immunosuppressive activity of myeloid-derived suppressor cells from prostate cancer patients. Clin Cancer Res 21: 3771-3782, 2015.

17. Yoon J, Ko YS, Cho SJ, Park J, Choi YS, Choi Y, Pyo JS, Ye SK, Youn HD, Lee JS, et al: Signal transducers and activators of transcription 3-induced metastatic potential in gastric cancer cells is enhanced by glycogen synthase kinase-3 $\beta$. APMIS 123: 373-382, 2015 .
18. Wang YX, Cai H, Jiang G, Zhou TB and Wu H: Silibinin inhibits proliferation, induces apoptosis and causes cell cycle arrest in human gastric cancer MGC803 cells via STAT3 pathway inhibition. Asian Pac J Cancer Prev 15: 6791-6798, 2014.

19. Silva IT, Carvalho A, Lang KL, Dudek SE, Masemann D, Durán FJ, Caro MS, Rapp UR, Wixler V, Schenkel EP, et al: In vitro and in vivo antitumor activity of a novel semisynthetic derivative of cucurbitacin B. PLoS One 10: e0117794, 2015.

20. Li ZJ, Shin JM, Choi DK, Lim SK, Yoon TJ, Lee YH, Sohn KC, Im M, Lee Y, Seo YJ, et al: Inhibitory effect of cucurbitacin B on imiquimod-induced skin inflammation. Biochem Biophys Res Commun 459: 673-678, 2015.

21. Zhang M, Bian ZG, Zhang Y, Wang JH, Kan L, Wang X, Niu HY and He P: Cucurbitacin B inhibits proliferation and induces apoptosis via STAT3 pathway inhibition in A549 lung cancer cells. Mol Med Rep 10: 2905-2911, 2014.

22. Iwanski GB, Lee DH, En-Gal S, Doan NB, Castor B, Vogt M, Toh M, Bokemeyer C, Said JW, Thoennissen NH and Koeffler HP: Cucurbitacin B, a novel in vivo potentiator of gemcitabine with low toxicity in the treatment of pancreatic cancer. Br J Pharmacol 160: 998-1007, 2010.

23. Livak KJ and Schmittgen TD: Analysis of relative gene expression data using real-time quantitative PCR and the $2^{-\Delta \Delta \mathrm{Ct}}$ method. Methods 25: 402-408, 2001.

24. Xu W and McArthur G: Cell cycle regulation and melanoma. Curr Oncol Rep 18: 34, 2016.

25. Yan YB: Creatine kinase in cell cycle regulation and cancer. Amino acids 48: 1775-1784, 2016.

26. Jirawatnotai S, Aziyu A, Osmundson EC, Moons DS, Zou X, Kineman RD and Kiyokawa $\mathrm{H}$ : Cdk4 is indispensable for postnatal proliferation of the anterior pituitary. J Biol Chem 279: 51100-51106, 2004.

27. Martín A, Odajima J, Hunt SL, Dubus P, Ortega S, Malumbres M and Barbacid M: Cdk2 is dispensable for cell cycle inhibition and tumor suppression mediated by p27 (Kip1) and p21 (Cip1). Cancer Cell 7: 591-598, 2005.

28. Abukhdeir AM and Park BH: P21 and p27: Roles in carcinogenesis and drug resistance. Expert Rev Mol Med 10: e19, 2008.

29. Kiraz Y, Adan A, Kartal Yandim M and Baran Y: Major apoptotic mechanisms and genes involved in apoptosis. Tumour Biol 37: 8471-8486, 2016

30. Monian $\mathrm{P}$ and Jiang $\mathrm{X}$ : Clearing the final hurdles to mitochondrial apoptosis: Regulation post cytochrome $\mathrm{C}$ release. Exp Oncol 34: 185-191, 2012.

31. Huber HJ, Duessmann H, Wenus J, Kilbride SM and Prehn JH: Mathematical modelling of the mitochondrial apoptosis pathway. Biochim Biophys Acta 1813: 608-615, 2011.

32. Kanai M, Konda Y, Nakajima T, Izumi Y, Kanda N, Nanakin A, Kubohara Y and Chiba T: Differentiation-inducing factor-1 (DIF-1) inhibits STAT3 activity involved in gastric cancer cell proliferation via MEK-ERK-dependent pathway. Oncogene 22: 548-554, 2003.

33. Yu LF, Cheng Y, Qiao MM, Zhang YP and Wu YL: Activation of STAT3 signaling in human stomach adenocarcinoma drug-resistant cell line and its relationship with expression of vascular endothelial growth factor. World J Gastroenterol 11: 875-879, 2005.

34. Schauer M, Janssen KP, Rimkus C, Raggi M, Feith M, Friess H and Theisen J: Microarray-based response prediction in esophageal adenocarcinoma. Clin Cancer Res 16: 330-337, 2010.

35. Yu LF, Zhu YB, Qiao MM, Zhong J, Tu SP and Wu YL: Constitutive activation and clinical significance of Stat3 in human gastric cancer tissues and cell lines. Zhonghua Yi Xue Za Zhi 84: 2064-2069, 2004 (In Chinese)

36. Thoennissen NH, Iwanski GB, Doan NB, Okamoto R, Lin P, Abbassi S, Song JH, Yin D, Toh M, Xie WD, et al: Cucurbitacin B induces apoptosis by inhibition of the JAK/STAT pathway and potentiates antiproliferative effects of gemcitabine on pancreatic cancer cells. Cancer Res 69: 5876-5884, 2009

37. Zheng Q, Liu Y, Liu W, Ma F, Zhou Y, Chen M, Chang J, Wang Y, Yang G and He G: Cucurbitacin B inhibits growth and induces apoptosis through the JAK2/STAT3 and MAPK pathways in SH-SY5Y human neuroblastoma cells. Mol Med Rep 10: 89-94, 2014. 